\title{
3D Reconstruction and Separation of Nickel and Zirconia Based Phases from Solid Oxide Fuel Cell Anode Using Backscatter Electron Imaging
}

\author{
George Wetzel ${ }^{1}$, Taghi Darroudi ${ }^{1}$ and Laxmikant Saraf ${ }^{1}$ \\ ${ }^{1}$ Electron Microscopy Laboratory, Clemson University, Clemson SC 29634
}

Solid Oxide Fuel Cells (SOFC) are often viewed as one of the best electrochemically generated sources of electricity for high power applications. In all oxide SOFC, optimum ion transport is achieved though usage of complex oxides at the anode, electrolyte and cathode. Electron microscopy analysis of complex oxides often presents challenges due to the varying nature of dopants in these oxides. This often results in complications as a result of high temperature material sintering, where the possibility of phase transformations and diffusion strongly exist. In this study, our goal is to identify and separate complex oxide phases through advanced electron microscopy (EM) analysis in the bulk part of anode material.

Since typical EM analysis is a 2D technique, to study material properties in the bulk, systematic and controlled material removal followed by analysis on a newly exposed material surface is essential. High energy focused ion beam (FIB) sources are excellent tools that can remove material with good control over the depth [1]. In this work, we use a $40 \mathrm{kV} \mathrm{FIB} \mathrm{source} \mathrm{to} \mathrm{achieve} \mathrm{typical} \mathrm{100nm/cycle} \mathrm{of} \mathrm{anode}$ material removal followed by EM analysis on the newly exposed surface. We apply Hitachi's trademark procedure, 'mill-and-monitor,' using the Hitachi NB5000 nanoDUE'T FIB/SEM microscope [2]. Usage of a $40 \mathrm{kV}$ FIB source allowed us to achieve a smaller diameter FIB which resulted in a greater control over depth of material removal per milling cycle. EM analysis after each milling cycle of material removal was performed using the backscatter electron (BSE) detector. As is well known, BSE are high energy electrons as a result of elastic diffusion with no energy transfer. Generally, these are high angle electrons backscattering from Coulomb interactions with the nucleus without ionizing atoms from the sample material.

In all oxide anode supported-SOFC, the most popular anode is represented by a mixture of nickel oxide (NiO) and yttria-stabilized zirconia (YSZ) [3]. This is due to the fact that materials at the anode need to satisfy the requirement of either pure electronic conduction or an appropriate balance of mixed electronic/ionic conduction. $\mathrm{NiO}$ is a good catalyst and useful for hydrocarbon dissociation. In addition, $\mathrm{Ni}$ acts as an excellent source for electronic conduction. YSZ has a fluorite structure; oxygen vacancies in fluorite oxide structures can be increased by replacing a group 4 element with a transition metal (with matching ionic radii) either from the group 3 or lanthanoids category. For these reasons, our material at the anode is a mixture of $\mathrm{NiO}$ and $\mathrm{YSZ} . \mathrm{CeO}_{2}$ is used as an interface layer between $\mathrm{NiO}-\mathrm{YSZ}$ and YSZ to promote highly active reaction area boundaries with large surface area.

It is well known that the yield of secondary electrons (SE) and BSE emission differ dramatically when the analyzed material contains high atomic number $(Z)$ elements. This difference is regularly used to achieve BSE image contrast between lighter and heavier elements. Therefore, such contrasts directly represent chemical differences in the material. Figure 1 indicates a graph representing yield / incident electron as a function of material atomic number Z. As shown in the graph, even though there is a minimal difference in the yield per incident electron between $\mathrm{Ni}(Z=28)$ and $\mathrm{Zr}(\mathrm{Z}=40)$ for the $\mathrm{SE}$ emission, the difference is substantial for BSE yield per incident electron. 
Figure 2 (a-c) shows a 3D reconstruction of the SOFC anode where, based on BSE imaging contrast differences, grains representing $\mathrm{Zr}$ and $\mathrm{Ni}$ are separated (Figures a\&b). Figure 2 (c) shows combined $\mathrm{Ni}+\mathrm{Ce}$ grains with some offset. Figure 3 shows X-ray dot maps from the NiO-YSZ anode and $\mathrm{CeO}_{2}$ interface using ED. During phase analysis, it was observed that a mixture of $\mathrm{Ce}$ and Ni exists at the YSZ, interface indicating $\mathrm{Ni}$ diffusion. We will discuss effects of $\mathrm{Ni}$ diffusion and experimental parameters used in this study on the functionality of SOFC.

References:

[1] J.R. Michael, Microscopy and Microanalysis, 17 (2011), p.386.

[2] Hitachi product manual, nanoDUE'T FIB-SEM NB5000, Web: http://www.hitachi-

hightech.com/us/product_detail/?pn=em-nb5000

[3] L.V. Saraf, D Baer, A Lea, et. al, J. Electrochem Soc., 157 (2010), p.B463.

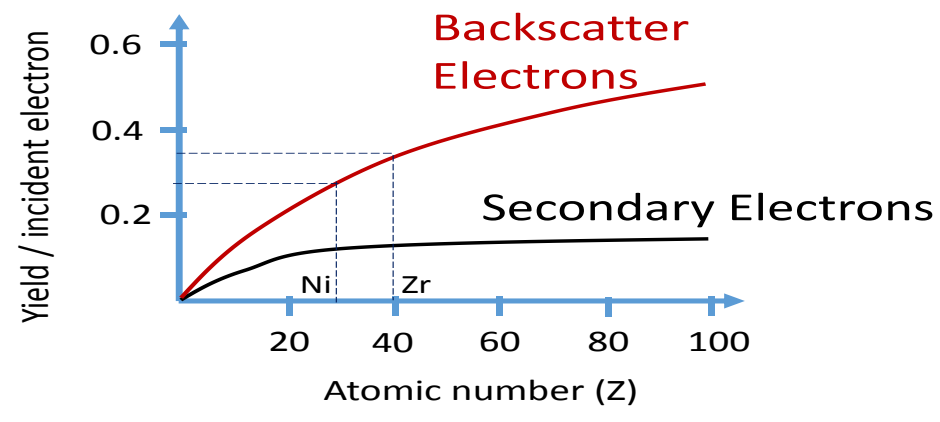

Figure 1. Yield per incident electron as a function of atomic number. Backscatter electrons yield difference between $\mathrm{Ni}$ and $\mathrm{Zr}$ is measurable while analyzing differences in image contrasts.

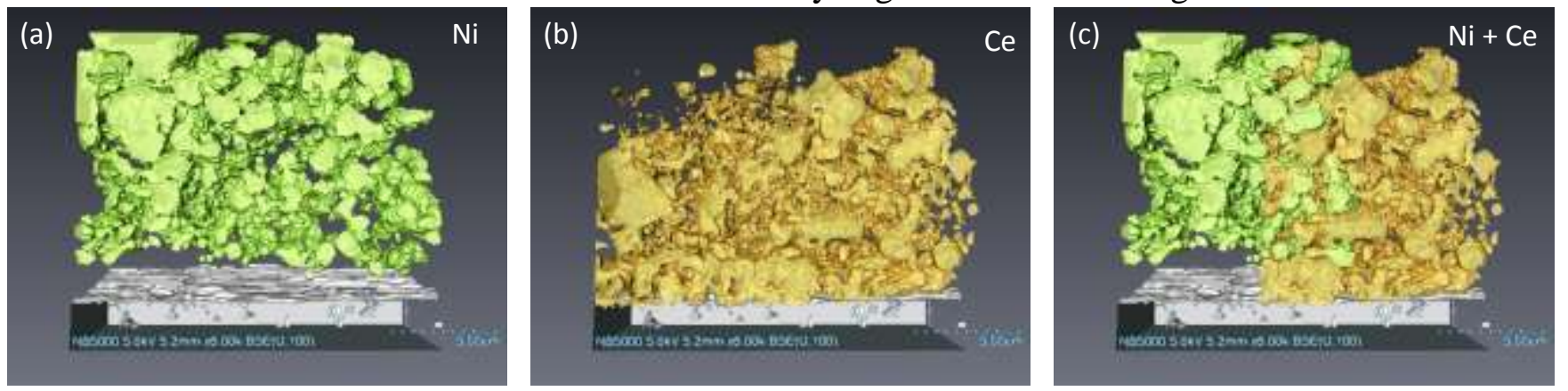

Figure 2. 3D reconstruction of (a) $\mathrm{Ni}$ (b) Ce and (c) Ni+Ce. BSE image contrast was used to identify the phase differences. For 3D analysis, $40 \mathrm{kV} \mathrm{FIB} \mathrm{with} \mathrm{Ga-ions} \mathrm{along} \mathrm{with} \mathrm{BSE} \mathrm{imaging} \mathrm{were} \mathrm{used} \mathrm{on}$ newly created surfaces at a separation of $100 \mathrm{~nm}$.

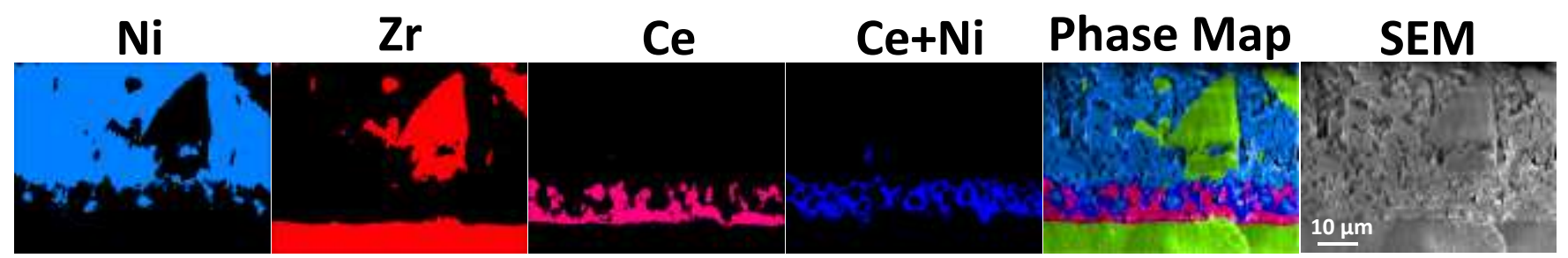

Figure 3. X-ray dot maps from NiO-YSZ anode and $\mathrm{CeO}_{2}$ interface using EDS. A phase mixture of $\mathrm{Ce}+\mathrm{Ni}$ is clearly observed at YSZ interface indicating diffusion of nickel. 